\title{
The anatomical safe zone for medial opening oblique wedge high tibial osteotomy
}

\author{
Bavornrat Vanadurongwan ${ }^{1}$,MD, Thana Siripisitsak ${ }^{1}$, MD, Narumol Sudjai ${ }^{1}$, BSc, Thossart Harnroongroj $^{1}$, MD
}

INTRODUCTION The end of medial opening oblique wedge high tibial osteotomy (HTO) points into a narrow area between the articular cartilage of the posterolateral proximal tibia and proximal tibiofibular joint (PTFJ) at the tibial site, which is an anatomical safe zone (ASZ) for osteotomy. We studied the ASZ and its relation to the fibular tip, including the level of posterior cruciate ligament $(\mathrm{PCL})$ insertion, to avoid penetration into the knee and PTFJ, and PCL injury by osteotomy.

METHODS Ten pairs of embalmed cadaveric legs were disarticulated at the knee joint and then examined. Soft tissues at the proximal tibia were removed. The posterior capsule of the PTFJ was incised to identify the articular cartilage of the PTFJ at the tibial site. The height of the fibular tip and the thickness of the ASZ were measured and calculated to determine the relationship between the ASZ and fibular tip. The level of PCL insertion was measured from the posterior articular surface of the proximal tibia to the distal attachment of the PCL.

RESULTS The average height of the fibular tip and the thickness of the ASZ were $5.43 \pm 1.53 \mathrm{~mm}$ and $4.12 \pm 1.60 \mathrm{~mm}$, respectively. On average, the fibular tip was $1.31 \pm 1.28 \mathrm{~mm}$ higher than the ASZ, and the level of PCL insertion was $10.10 \pm 1.88 \mathrm{~mm}$.

CONCLUSION To ensure safety during medial opening oblique wedge HTO, the end of osteotomy should point accurately into the ASZ at a level just below the fibular tip as reference. Proximal osteotomy thickness should not be less than $10 \mathrm{~mm}$ at the level of PCL insertion.

Keywords: anatomical safe zone, fibular tip, medial opening oblique wedge high tibial osteotomy, posterior cruciate insertion

Singapore Med J 2013; 54(2): 102-104

\section{INTRODUCTION}

Medial oblique opening wedge valgus high tibial osteotomy (HTO) above the fibula is a common realignment procedure for treating medial unicompartment osteoarthritic knee. ${ }^{(1-4)}$ This technique is usually performed with an oblique cut starting from the medial to the lateral cortex of the proximal tibia. Adequate thickness of osteotomy is necessary for internal fixation and avoids posterior cruciate ligament $(\mathrm{PCL})$ insertion injury. The location of end osteotomy at the lateral cortex of the tibial condyle must be between the lower end of the posterolateral articular cartilage of the proximal tibia and the proximal tibiofibular joint (PTFJ). This narrow area is often called the anatomical safe zone (ASZ) for medial oblique opening wedge HTO. ${ }^{(5,6)}$ Penetration into the articular cartilage of the lateral tibial condyle or PTFJ is a common complication during osteotomy. ${ }^{(7-9)}$ Moreover, inadequate thickness of the proximal osteotomy can result in the risk of PCL insertion injury and the problem of stable internal fixation. Understanding the ASZ at the posterolateral proximal tibia and its relationship to the fibular tip, including the appropriate thickness of the proximal osteotomy, may help surgeons preclude such complications. This study was carried out to determine the thickness of the ASZ, including its relationship to the fibular tip, and the level of PCL insertion at the proximal tibia.

\section{METHODS}

Ten pairs of embalmed, normal cadaveric legs containing intact PTFJs were included in the study (age 48-60 years). All specimens were disarticulated at the level of the knee joint. Soft tissues around the proximal tibia and PTFJ were dissected to identify the articular cartilage of the tibial condyles and PCL insertion. The posterior capsule of the PTFJ was longitudinally incised to identify the articular surface at the tibial site. Incisions were made carefully in order to avoid causing instability of the PTFJ. Using a digital vernier calliper, the height of the fibular tip was determined as the distance from the fibular tip to the upper end of the articular cartilage of the PTFJ at the tibial site (Fig. 1). The capsule of the PTFJ was then completely excised. The fibular head was displaced anteriorly and the posterolateral aspect of the proximal tibia exposed. The ASZ for osteotomy was identified as the area between the end of the articular cartilage of the posterolateral corner of the proximal tibia and the upper articular cartilage of the PTFJ at the tibial site. The thickness of the ASZ was then measured (Fig. 2). The distance between the ASZ and the fibular tip was calculated. The level of PCL insertion was measured as the distance from the end of the posterior articular surface of the proximal tibial to the lowest insertion of the PCL. Tibial and fibular lengths were measured using a ruler. The data were presented as

${ }^{1}$ Department of Orthopaedic Surgery, Siriraj Hospital, Mahidol University, Bangkok, Thailand

Correspondence: Prof Thossart Harnroongroj, Professor, Department of Orthopaedics Surgery, Faculty of Medicine, Siriraj Hospital, Mahidol University, Bangkok 10700, Thailand. tmthr@mahidol.ac.th 


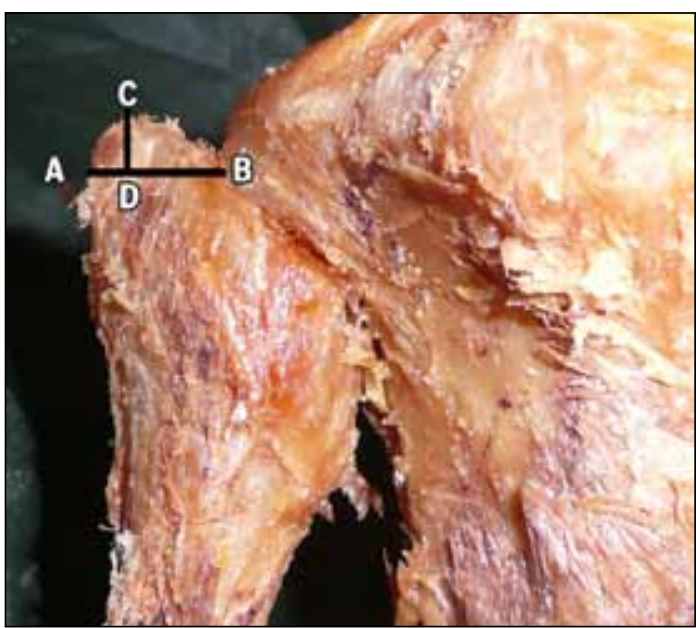

Fig. 1 Photograph shows the measurement of the height of the fibular tip (CD). AB: horizontal level of the upper end of the articular cartilage of the PTFJ at the tibial site; C: fibular tip; D: the point where $C D$ is perpendicular to $A B$.

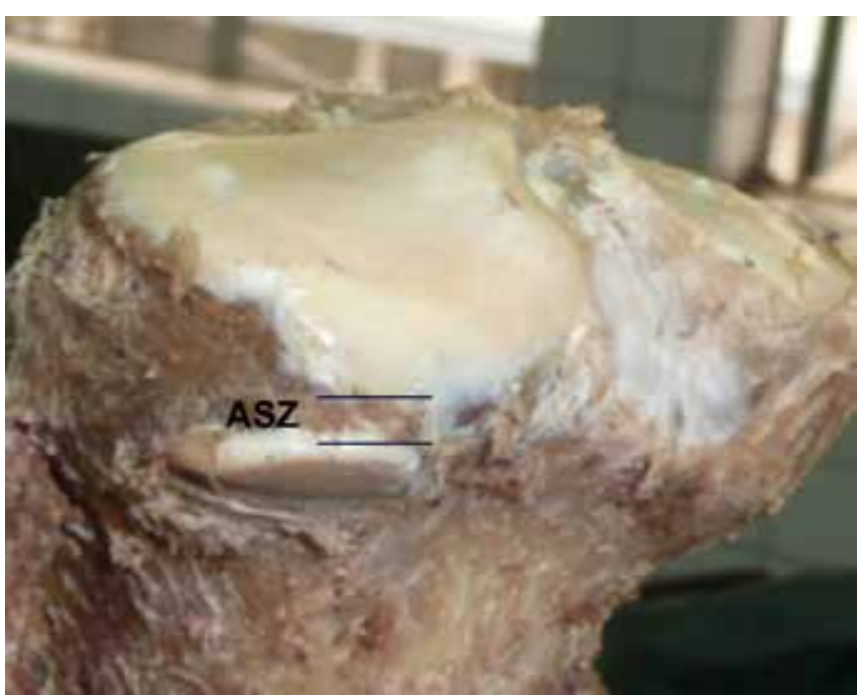

Fig. 2 Photograph shows the measurement of the thickness of the anatomical safe zone (ASZ).

Table I. Study findings based on cadaveric measurements of the anatomical safe zone.

\begin{tabular}{|c|c|c|c|c|c|c|}
\hline Specimen no. & $\begin{array}{c}\text { Thickness of } \\
\text { ASZ (mm) }\end{array}$ & $\begin{array}{c}\text { Fibular height } \\
\text { (mm) }\end{array}$ & $\begin{array}{l}\text { Relationship of ASZ } \\
\text { and fibular tip ( } \mathrm{mm})\end{array}$ & $\begin{array}{c}\text { PCL insertion } \\
(\mathrm{mm})\end{array}$ & $\begin{array}{l}\text { Tibial length } \\
(\mathrm{mm})\end{array}$ & $\begin{array}{l}\text { Fibular length } \\
\text { (mm) }\end{array}$ \\
\hline 1 & 4.29 & 5.56 & 1.27 & 9.0 & 355 & 360 \\
\hline 2 & 4.88 & 3.65 & -1.23 & 8.5 & 355 & 360 \\
\hline 3 & 5.12 & 5.32 & 0.20 & 8.0 & 330 & 335 \\
\hline 4 & 7.15 & 8.11 & 0.96 & 10.0 & 330 & 335 \\
\hline 6 & 4.54 & 3.98 & -0.56 & 10.0 & 340 & 360 \\
\hline 7 & 2.09 & 4.67 & 2.58 & 9.5 & 300 & 325 \\
\hline 8 & 3.19 & 5.88 & 2.69 & 11.5 & 300 & 325 \\
\hline 9 & 4.60 & 5.41 & 0.81 & 6.5 & 360 & 375 \\
\hline 10 & 5.07 & 4.31 & -0.76 & 8.0 & 360 & 375 \\
\hline 12 & 4.01 & 6.53 & 2.52 & 11.0 & 335 & 350 \\
\hline 13 & 3.55 & 7.38 & 3.83 & 9.0 & 335 & 350 \\
\hline 14 & 3.31 & 5.25 & 1.94 & 11.0 & 335 & 350 \\
\hline 15 & 6.85 & 7.59 & 0.74 & 12.0 & 340 & 360 \\
\hline 16 & 4.70 & 5.66 & 0.96 & 11.5 & 340 & 360 \\
\hline 17 & 2.78 & 3.48 & 0.70 & 9.5 & 290 & 300 \\
\hline 18 & 0.54 & 2.41 & 1.87 & 11.0 & 290 & 300 \\
\hline 19 & 2.58 & 4.43 & 1.85 & 14.0 & 375 & 385 \\
\hline 20 & 3.59 & 5.18 & 1.59 & 13.5 & 375 & 385 \\
\hline Mean measurement* & $\begin{array}{l}4.12 \pm 1.60 \\
(3.37-4.87)\end{array}$ & $\begin{array}{l}5.43 \pm 1.53 \\
(4.71-6.14)\end{array}$ & $\begin{array}{l}1.31 \pm 1.28 \\
(0.71-1.91)\end{array}$ & $\begin{array}{c}10.10 \pm 1.88 \\
(9.22-10.98)\end{array}$ & $\begin{array}{c}336.00 \pm 25.01 \\
(324.30-347.70)\end{array}$ & $\begin{array}{c}350.00 \pm 24.06 \\
(338.74-361.26)\end{array}$ \\
\hline
\end{tabular}

*Data is presented as mean \pm standard deviation $(95 \% \mathrm{Cl})$.

$\mathrm{Cl}$ : confidence interval; ASZ: anatomical safe zone; $\mathrm{PCL}$ : posterior cruciate ligament

mean \pm standard deviation, and the distance between the ASZ and the fibular tip was ascertained at $95 \%$ confidence interval $(\mathrm{Cl})$.

\section{RESULTS}

The average tibial and fibular lengths of the specimens were $336.00 \pm 25.01 \mathrm{~mm}(95 \% \mathrm{Cl} 324.30-347.70)$ and $350.00 \pm$ $24.06 \mathrm{~mm}$ (95\% Cl 338.74-361.26), respectively. The average height of the fibular tip was $5.43 \pm 1.53 \mathrm{~mm}(95 \% \mathrm{Cl} 4.71-6.14)$, and the average thickness of the ASZ was $4.12 \pm 1.60 \mathrm{~mm}(95 \%$ $\mathrm{Cl}$ 3.37-4.87). On average, the distance between the ASZ and the fibular tip was determined as $1.31 \pm 1.28 \mathrm{~mm}(95 \% \mathrm{Cl} 0.71-1.91)$ above the ASZ. In 17 (85\%) specimens, the tip of the fibula was $1.69 \pm 0.95 \mathrm{~mm}$ above the ASZ, while in three (15\%) specimens, it was $0.86 \pm 0.33$ within the level of the ASZ. The average PCL insertion was $10.10 \pm 1.88 \mathrm{~mm}(95 \% \mathrm{Cl} 9.22-10.98)$ below the posterior articular surface of the proximal tibia (Table I). 


\section{DISCUSSION}

Medial oblique opening wedge valgus $\mathrm{HTO}$, recommended as a surgical technique for the treatment of medial unicompartment osteoarthritic knee, provides high success rates for an extended time period. ${ }^{(1-4)}$ In 1965, Coventry performed HTO at the level of a previous epiphyseal line and just proximal to the tibial tubercle using the lateral closed wedge technique with the upper portion of the fibula removed. ${ }^{(10)}$ The calculated corrected angle plus additional degrees of correction were obtained by lateral bone removal. The region of osteotomy was immobilised by cylindrical cast until healing. ${ }^{(10)}$ Lateral closing wedge osteotomy was further developed in terms of osteotomy cut, and stable fixation was obtained by using special plate fixation. ${ }^{(11)}$ However, fibular osteotomy was always necessary for this technique. Various medial opening wedge HTO techniques without fibular osteotomy were developed to achieve adequate thickness of the proximal osteotomy for stable internal fixation and to control corrected angle without cutting out bone. . $2,3,12)^{-}$

At present, most surgeons perform osteotomy by using medial oblique opening wedge HTO above the fibula. ${ }^{(12)}$ However, this technique has complications due to penetration into the articular cartilage of the lateral tibial condyle or into the PTFJ. ${ }^{(7-9)}$ The ASZ for the lateral end of osteotomy is narrow and located between the posterolateral articular cartilage of the proximal tibia and PTFJ. ${ }^{(5,6,13)}$ As shown in our study, the mean thickness of the ASZ was only $4.12 \mathrm{~mm}$. It is therefore vital that the osteotomy must be accurately pointed into the ASZ. Most surgeons prefer osteotomy below the joint line of the knee, although the osteotomy may penetrate into the PTFJ. However, the perfect osteotomy shoulder is when the end osteotomy is between the articular cartilage of the lateral tibial condyle and PTFJ. Intraoperatively, the fibular tip is routinely used as an anatomical landmark for directing the end of the osteotomy into the ASZ. Nevertheless, our study showed that the fibular tip was on average $1.69 \mathrm{~mm}$ above the ASZ in most of the specimens studied (even though analysis by case showed that $15 \%$ of the fibular tips were within the ASZ). This implies that the direction of safe osteotomy line should be just below the fibular tip instead of at the same level.

The appropriate thickness of proximal osteotomy is important. Greater thickness provides for stable fixations, but as the osteotomy is near the diaphyses, there may be problems with bone healing. On the other hand, proximal osteotomy that is too thin may present problems such as stable internal fixation with a likelihood of PCL insertion injury. As shown in this study, a safe thickness of proximal osteotomy should not be less than $10 \mathrm{~mm}$ at the level of PCL insertion. However, tibial attachment of the PCL is a vast area of study and our attempt did not evaluate whether injury at this level created PCL insufficiency.

In conclusion, we recommend that medial oblique opening wedge valgus $\mathrm{HTO}$ is safe so long as the lateral end of the osteotomy is accurately pointing into the ASZ, using the level just below the fibular tip as the reference marker. The level of the fibular tip was found to be unsafe as a definite reference point for osteotomy. The proximal thickness of the osteotomy should be at least $10 \mathrm{~mm}$ at the level of PCL insertion. Further studies on the intraoperative radiographic assessment of the ASZ would be of more practical use to surgeons performing medial oblique opening wedge HTO.

\section{REFERENCES}

1. Harnroongroj T, Vanadurongwan V. The stable upper tibial osteotomy for the treatment of osteoarthritis knee: lateral closing step wedge osteotomy (Part I). Siriraj Med J 1993; 45:829-36

2. Harnroongroj T, Vanadurongwan $\mathrm{V}$. The stable upper tibial osteotomy for the treatment of osteoarthritic knee: Medial opening step wedge technique (Part II). Siriraj Med J 1994; 46:28-33.

3. Harnroongroj $T$, Vanadurongwan $\mathrm{V}$. The stable upper tibial osteotomy for treatment of osteoarthritic knee (Part III): the simplified medial opening step wedge technique. Siriraj Med J 1994; 46:120-5.

4. Schallberger A, Jacobi M, Wahl P, Maestretti G, Jakob RP. High tibial valgus osteotomy in unicompartmental medial osteoarthritis of the knee: a retrospective follow-up study over 13-21 years. Knee Surg Sports Traumatol Arthrosc 2011; 19:122-7.

5. Bozkurt M, Yilmaz E, Atlihan D, et al. The proximal tibiofibular joint: an anatomic study. Clin Orthop Relat Res 2003; 406:136-40.

6. Eichenblat $M$, Nathan $H$. The proximal tibiofibular joint. An anatomical study with clinical and pathological considerations. Int Orthop 1983; 7:31-9.

7. Song EK, Seon JK, Park SJ, Jeong MS. The complications of high tibial osteotomy: closing-versus opening wedge method. J Bone Joint Surg $\mathrm{Br}$ 2010; 92:1245-52.

8. Spahn G. Complications in high tibial (medial opening wedge) osteotomy. Arch Orthop Trauma Surg 2004; 124:649-53.

9. Esenkaya I, Elmali N, Kaygusuz MA, Misirlioğlu M, Atasever A. [Assessment of the vulnerability of the proximal tibiofibular joint to injury during osteotomies]. Acta Orthop Traumatol Turc 2006; 40:396-402. Turkish.

10. Coventry MB. Osteotomy of the upper portion of the tibia for degenerative arthritis of the knee. J Bone Joint Surg Am 1965; 47:984-90.

11. Coventry MB. Upper tibial osteotomy for osteoarthritis. J Bone Joint Surg Am 1985; 67:1136-40.

12. Franco V, Cerullo G, Cipolla M, et al. Open wedge high tibial osteotomy. Tech Knee Surg 2002 2002; 1:43-53.

13. Ogden JA. The anatomy and function of the proximal tibiofibular joint. Clin Orthop Relat Res 1974; 101:186-91. 